\title{
OPEN Cryopreservation of Anopheles stephensi embryos
}

\author{
Eric R. James ${ }^{1 \bowtie}$, Yingda Wen ${ }^{1}$, James Overby ${ }^{1}$, Kristen Pluchino ${ }^{1}$, Shane McTighe ${ }^{1,2}$, \\ Stephen Matheny ${ }^{1}$, Abraham Eappen ${ }^{1}$, Stephen L. Hoffman ${ }^{1}$ \& Peter F. Billingsley ${ }^{1}$
}

The ability to cryopreserve mosquitoes would revolutionize work on these vectors of major human infectious diseases by conserving stocks, new isolates, lab-bred strains, and transgenic lines that currently require continuous life cycle maintenance. Efforts over several decades to develop a method for cryopreservation have, until now, been fruitless: we describe here a method for the cryopreservation of Anopheles stephensi embryos yielding hatch rates of $\sim 25 \%$, stable for $>5$ years. Hatched larvae developed into fertile, fecund adults and blood-fed females, produced fully viable second generation eggs, that could be infected with Plasmodium falciparum at high intensities. The key components of the cryopreservation method are: embryos at 15-30 min post oviposition, two incubation steps in $100 \%$ deuterated methanol at $-7^{\circ} \mathrm{C}$ and $-14.5^{\circ} \mathrm{C}$, and rapid cooling. Eggs are recovered by rapid warming with concomitant dilution of cryoprotectant. Eggs of genetically modified A. stephensi and of $A$. gambiae were also successfully cryopreserved. This enabling methodology will allow long-term conservation of mosquitoes as well as acceleration of genetic studies and facilitation of mass storage of anopheline mosquitoes for release programs.

Maintenance of Anopheles requires continuous culture of the mosquito life cycle, a process that is labor intensive, expensive and vulnerable to colony loss. Until now, no stage of the Anopheles life cycle has been preserved; adults can be held for 3-4 weeks ${ }^{1}$ with low survival, while egg survival is limited to 1-10 days depending on humidity and temperature $e^{2,3}$.

The goal of this study was to develop a method for cryopreservation of Anopheles eggs for the purpose of eventually creating a characterized egg bank under current good manufacturing practice (cGMP) for use in the manufacture of Sanaria PfSPZ vaccines against malaria ${ }^{4-9}$. The method was also intended to allow banking of new isolates, strains and transgenic lines to aid research on mosquito genetics, malaria and other mosquitotransmitted diseases.

\section{Materials and methods}

Mosquito embryo collection. Anopheles stephensi SDA 500 mosquitoes ${ }^{10}$ have been maintained at Sanaria since 2005 and designated internally as A. stephensi SDA500 (9800). Mosquitoes were fed through an artificial membrane on human O + blood (Mid-South Blood Bank, Memphis, TN) by standard methods and housed in $300 \times 300 \times 300 \mathrm{~mm}$ cages $^{11}$. Three days post-feeding, a $150 \mathrm{~mm}$ diameter Petri dish $(25384-118$, VWR) containing $\sim 75 \mathrm{~mL}$ tap water was introduced into the cage and the mosquitoes were allowed to oviposit for $15 \mathrm{~min}$. Dead mosquitoes or mosquito fragments were removed. The floating eggs were swirled around the Petri dish so the majority adhered to the sides and lid, and the water was decanted from the dish. Eggs were collected using artists paint brushes (No. 4 black-tipped angular shader, MSPCI). Approximately 5,000 eggs could be collected on the upper side of one brush. Surface moisture was removed from the eggs immediately before transfer into cryoprotectant additive (CPA) by blotting the lower side of the brush on an absorbent paper towel.

Cryoprotectant additives (CPA). CPAs used were methanol (646377, Sigma-Aldrich) and D4-methanol (321281000, Acros Organics). CPA containers comprised polypropylene $19 \mathrm{~mm}$ outer diameter (OD) $\times 19 \mathrm{~mm}$ deep paint mixing pots (10271583, Michaels) housed in $19 \mathrm{~mm}$ internal diameter (ID) $\times 15 \mathrm{~mm}$ deep holes milled into a custom aluminium block (see supplementary information, F1) machined to fit onto a Peltiercooled dual block apparatus (IC22XT, Torrey Pines Scientific). CPA for the first incubation step (2 mL) was equilibrated at $-7^{\circ} \mathrm{C}$ for $>1 \mathrm{~h}$ prior to addition of eggs. After the first incubation of eggs in $\mathrm{CPA}$ at $-7^{\circ} \mathrm{C}$, the container of CPA with eggs was transferred to a second custom aluminium block (see supplementary information, F1) with 3 holes to accept CPA containers similarly supported on a second Peltier-cooled dual block

${ }^{1}$ Sanaria Inc, Rockville, MD, USA. ${ }^{2}$ Department of Dermatology, Walter Reed National Military Medical Center, Rockville, MD, USA. ${ }^{\varpi}$ email: ejames@sanaria.com 
apparatus (IC22XT, Torrey Pines Scientific) at $-14.5^{\circ} \mathrm{C}$. This block also had three $12 \mathrm{~mm}$ wide steps running the length of the block on which the rectangular card stock supports were placed at right angles with one end extending beyond the step.

Sample supports. Card supports for the aliquots of eggs suspended in CPA were cut from $0.14 \mathrm{~mm}$ thick black card stock into $15 \mathrm{~mm} \times 5 \mathrm{~mm}$ rectangles. These card supports were permeable to CPA and relatively rigid but thin enough to facilitate rapid cooling. The black color of the supports provided contrast for easy visualization of the eggs which are white before melanization begins.

Samples. The card supports were placed on the steps of the cold block at $-14.5^{\circ} \mathrm{C}$. At the end of the second incubation step at $-14.5^{\circ} \mathrm{C}, 20 \mu \mathrm{L}$ aliquots of eggs in CPA were transferred using a P200 pipettor (Ranin) with truncated tip (ID $1.5 \mathrm{~mm}$ ) to the chilled card supports. Aliquots were allowed to spread on the cards and the CPA to be partially absorbed to reduce surface thickness and improve direct contact of the eggs with the liquid nitrogen $\left(\mathrm{LN}_{2}\right)$ during cooling. Aliquots of eggs were transferred to the card supports $10-30 \mathrm{~s}$ before the final cooling step into $\mathrm{LN}_{2}$ commenced when the $15 \mathrm{~min}$ incubation in CPA at $-14.5^{\circ} \mathrm{C}$ was completed.

Cooling and storage. The refrigerant for cooling was either boiling point $\mathrm{LN}_{2}$ at $-196{ }^{\circ} \mathrm{C}$, or chilled $\mathrm{LN}_{2}$ between -202 and $-210^{\circ} \mathrm{C}$ that was produced by applying a vacuum source to $\mathrm{LN}_{2}$ in a sealed insulated wide mouth vacuum flask $(70 \mathrm{~mm}$ ID $\times 110 \mathrm{~mm})$. Each card support with eggs was picked up by its free end using fine forceps and immediately transferred directly into the $\mathrm{LN}_{2}$ refrigerant. Each card support was held under the surface of the $\mathrm{LN}_{2}$ for $\sim 3 \mathrm{~s}$ and then transferred to temporary storage in a 6-hole aluminium block (10055-264, VWR) in $\mathrm{LN}_{2}$ in an insulated container. Card supports were then transferred into cryovials (Nunc $2.0 \mathrm{~mL}$ ), previously chilled in $\mathrm{LN}_{2}$, and then stored in an isothermal liquid nitrogen vapor phase (LNVP) freezer (V1500AB, Custom Biogenic System).

Thawing and dilution. To test the larval hatch rates from cryopreserved eggs, sample aliquots on card supports were thawed individually from $\mathrm{LN}_{2}$. Thawing and CPA dilution were performed in a single step by rapid transfer of each card support into a stream of water at $23^{\circ} \mathrm{C}$ from a $500 \mathrm{~mL}$ wash bottle (414004-227, VWR) to a final volume of $10 \mathrm{~mL}$ collected into a $50 \mathrm{~mm}$ diameter Petri dish (25384-322, VWR). Petri dishes were placed on trays in an incubator (Sanyo) at $28^{\circ} \mathrm{C}$ for chorion melanization, embryo development and larval hatching.

Survival assessment. On day 1 post-thaw, samples in the Petri dishes contained a mix of white non-viable and dark putatively viable eggs. The proportion of dark eggs was used initially to assess the tolerance of eggs to different CPAs (including ethanediol, propanediol, dimethyl sulphoxide, and glycerol), different CPA concentrations, and cooling and warming parameters. Survival assessment: Experimental conditions for cryopreservation were developed with melanization as the marker until some eggs developed into embryos with eye spots by days 2-3 post thawing and the first hatched larvae were obtained. Once the first larval hatches had been recorded, the proportion of eggs hatching (number of larvae $\times 100 /$ number of eggs in sample) on day 3 post thaw was used as the measure of survival. In experiments where full development was assessed, hatched larvae were fed standard larval food (Aqueon Betta fish food and Brewer's Yeast), pupae were harvested and transferred to bowls in $300 \times 300 \times 300 \mathrm{~mm}$ cages for adult mosquito emergence $\mathrm{e}^{11,12}$.

Infectivity assessment. The ability of mosquitoes to be infected with Plasmodium falciparum (Pf) was determined by feeding mosquitoes through an artificial membrane on blood cultures containing $0.5 \%$ stage $\mathrm{V}$ Pf gametocytes ${ }^{12}$.

\section{Results and discussion}

The chitin-rich chorion of anopheline eggs ${ }^{13}$ is a barrier to water movement out of the embryo and to the entry of CPAs, and methods to increase permeability have been the main focus of previous studies aimed at cryopreservation $^{14-16}$. Drosophila melanogaster eggs were successfully cryopreserved only after treatment with benzalkonium chloride and heptane to allow permeation of the two CPAs, glycerol and ethanediol, across the egg chorion ${ }^{17,18}$. This same treatment was lethal for Anopheles eggs: the chorion structure, embryo tegument, and permeability to water and CPAs ${ }^{19}$ are markedly different from Drosophila. However, at oviposition, Anopheles eggs are soft, non-sclerotized, and more readily water permeable ${ }^{20}$, attributes that decline as a function of embryo age.

Methanol, though not well tolerated by many cell types, is a highly effective CPA for a number of organisms, including protists ${ }^{21,22}$, trematodes ${ }^{23,24}$, nematodes ${ }^{25}$, and also fish spermatozoa and eggs ${ }^{26,27}$. Methanol, the lowest molecular weight CPA, appears to be the only CPA that can permeate Anopheles eggs, and outside of specific temperatures and with extended exposure times, it is toxic. At $-5^{\circ} \mathrm{C}$ few embryos survived a 15 -min exposure to $100 \%$ methanol and none survived temperatures above $-5^{\circ} \mathrm{C}$ (Fig. 1a). Nor did embryos survive a 15 min exposure to methanol at $-13^{\circ} \mathrm{C}$, in this case presumably due to ice formation within the embryos before the methanol could permeate sufficiently or before enough water had been removed. However, Anopheles embryos tolerated methanol at temperatures within these bounds with optimal recovery at $-11^{\circ} \mathrm{C}$ (Fig. 1a,b for non-cryopreserved eggs). Optimal survival using a single step incubation in methanol at $-11^{\circ} \mathrm{C}$ followed by plunge into $\mathrm{LN}_{2}$ was obtained after incubating the eggs for 20 min under which conditions $15.3 \pm 4.3 \%$ of the eggs hatched (Fig. $1 \mathrm{~b}$ dashed line).

The proportion of larvae that hatched from eggs cryopreserved using a single incubation temperature (Fig. 1b,e) was increased if the incubation in methanol was performed in two steps at two different temperatures 


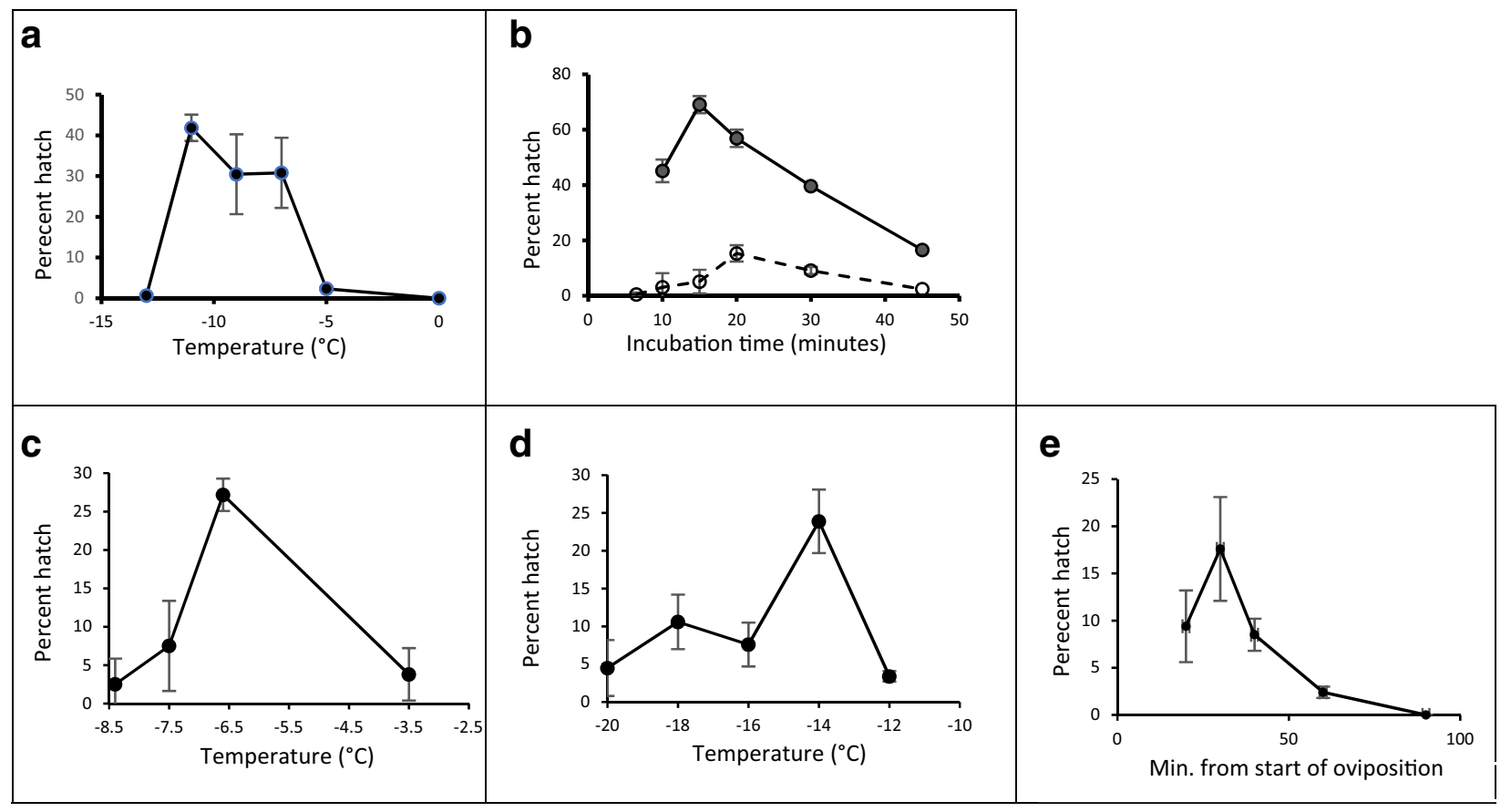

Figure 1. Parameters for oviposition and incubation in cryoprotectant additive (CPA: methanol) for $A$. stephensi egg cryopreservation. (a) Survival (percent hatch) of eggs following incubation in $100 \%$ methanol for $15 \mathrm{~min}$ at temperatures between 0 and $-13^{\circ} \mathrm{C}$ without subsequent cooling and with recovery into water at $23{ }^{\circ} \mathrm{C}$. (b) Survival (percent hatch) of eggs following incubation in $100 \%$ methanol for periods of $7-45 \mathrm{~min}$ at $-11^{\circ} \mathrm{C}$ and subsequent dilution into water at $23^{\circ} \mathrm{C}$. Eggs directly warmed/diluted from $-11^{\circ} \mathrm{C}$ without being rapidly cooled by plunging into $\mathrm{LN}_{2}$ (solid line); eggs incubated at $-11^{\circ} \mathrm{C}$ and subsequently plunged into $\mathrm{LN}_{2}$ before warming/dilution (dashed line). (c) Optimization of the incubation temperature for the first CPA incubation step with the second incubation step held constant at $-14.5^{\circ} \mathrm{C}$ for $15 \mathrm{~min}$. (d) Optimization of the incubation temperature for the second CPA incubation step with the first incubation step held constant at $-7^{\circ} \mathrm{C}$ for $6.5 \mathrm{~min}$. (e) Effect of oviposition time on survival: eggs were oviposited onto water in Petri dishes for $15 \mathrm{~min}$, harvested and then held an additional 5-75 min before adding to CPA (single incubation at $-11^{\circ} \mathrm{C}$ for $15 \mathrm{~min}$ ). The data points represent the age of the oldest eggs in each sample (20-90 min old).

(Fig. 1c,d), first at $-7^{\circ} \mathrm{C}$ for $6.5-7 \mathrm{~min}$ and subsequently at $-14.5^{\circ} \mathrm{C}$ for $15 \mathrm{~min}$. With this method, the partial dehydration and partial CPA permeation that occurs during the first incubation step allowed the eggs to survive cooling to below $-13^{\circ} \mathrm{C}$ (shown to be lethal in Fig. 1a), making further dehydration and methanol permeation possible during the extended second incubation step at $-14.5^{\circ} \mathrm{C}$. The toxicity of methanol is both temperature and time dependent, and the time that the eggs can tolerate immersion in methanol is extended by the lower temperature of the second incubation step.

The effect of the two incubation steps is to maximize the reduction in the amount of freezable intracellular water both through CPA-induced dehydration and by increasing the internal CPA concentration while minimizing CPA toxicity. These processes will combine to increase the viscosity of the intracellular contents enhancing their propensity to vitrify during rapid cooling in $\mathrm{LN}_{2}$. The results of experiments conducted to optimize the temperatures for the two incubation steps are shown in Fig. 1c,d.

At the end of the second incubation step, the eggs dispensed in $20 \mu \mathrm{L}$ aliquots on card supports and held at $-14.5^{\circ} \mathrm{C}$, were plunged directly into boiling point $\mathrm{LN}_{2}$ (cooling rate of $-12,240 \pm 151{ }^{\circ} \mathrm{C} \mathrm{min}^{-1}$ ) or into $\mathrm{LN}_{2}$ chilled to $-202{ }^{\circ} \mathrm{C}$ or below (cooling rate $-87,864 \pm 6315^{\circ} \mathrm{C} \mathrm{min}^{-1}$ ).

In twelve experiments comparing boiling point vs chilled $\mathrm{LN}_{2}$, hatch rates were marginally, but not statistically significantly, higher for samples cooled using chilled $\mathrm{LN}_{2}$. Similarly, a small, but not statistically significant increase in hatch rate was obtained using D4-deuterated methanol which has an approximately $10.7 \%$ higher viscosity than $\mathrm{H} 4$-methanol $\left(\text { at } 25^{\circ} \mathrm{C}\right)^{28}$.

Following storage in LNVP below $-150^{\circ} \mathrm{C}$, recovery was accomplished through simultaneous thawing and dilution to remove CPA by holding the card support with eggs under a stream of water directed into a Petri dish. This operation was performed rapidly. The optimal water temperature was $23^{\circ} \mathrm{C}$ (data not shown) which



Embryos of most species cryopreserve optimally when cells are undifferentiated ${ }^{29,30}$, when the embryos are potentially able to tolerate the loss of some proportion of their cells without subsequent development being impaired. Counterintuitively, successful cryopreservation of Drosophila eggs was optimal at $12-15 \mathrm{~h}$ post oviposition ${ }^{17,18}$ when embryo development is nearly complete. Anopheles eggs are fertilized at oviposition, and the embryo develops as a syncytium until cell wall involution which becomes visible at approximately $4-6 \mathrm{~h}^{16}$. A. stephensi eggs require $48-72 \mathrm{~h}$ at $28^{\circ} \mathrm{C}$ for larval emergence. By harvesting Anopheles eggs soon after oviposition when the eggshell is soft ${ }^{16,19}$ and relatively porous to water and methanol, there is no need for permeabilization. 


\begin{tabular}{|l|l|l|}
\hline & First generation & Second generation \\
\hline Number of mosquitoes dissected & 6 & 30 \\
\hline Prevalence of PfSPZ infection (percent mosquitoes infected) & $50 \%$ & $93 \%$ \\
\hline Intensity of infection (mean PfSPZ/mosquito) & 30,833 & 57,835 \\
\hline Mean PfSPZ per infected mosquito & 61,667 & 62,188 \\
\hline Range of PfSPZ per infected mosquito & $49,000-78,000$ & $250-276,500$ \\
\hline
\end{tabular}

Table 1. P. falciparum infection of adult A. stephensi derived from cryopreserved eggs. Adult mosquitoes grown from cryopreserved eggs and second-generation adults reared from eggs laid by those first generation cryopreserved mosquitoes were fed on cultured stage V Pf gametocytes, and the numbers of sporozoites (PfSPZ) in the salivary glands were determined 14 days later.

Maximum survival of eggs exposed to $100 \%$ methanol at $-11{ }^{\circ} \mathrm{C}$ for $15 \mathrm{~min}$ and cooled to $-196^{\circ} \mathrm{C}$ occurred at 15-30 min after oviposition (Fig. 1e). The subsequent decline may be related to the progressive chemical hardening of the chorion affecting permeability and/or to changes in the developing embryo, including the early stages of cellular differentiation.

After storage in LNVP for 24 and 60 months, hatch rates were $28.2 \pm 5.5 \%$ and $23.3 \pm 2.0 \%$, respectively. Cryopreserved eggs thawed and subsequently maintained at $28{ }^{\circ} \mathrm{C}$ in water hatched on days 2 and 3 , the same timing as control (not cryopreserved) eggs, and the development time of larvae through to pupation followed a similar timeline for cryopreserved and control eggs. The proportion of hatched larvae derived from cryopreserved eggs that subsequently pupated was $76.3 \%$, and of these pupae $87 \%$ yielded adult mosquitoes of which $49.7 \%$ were females.

The feeding behavior of adult mosquitoes derived from cryopreserved eggs was unaffected by cryopreservation (Table 1). The rate of development of mosquitoes obtained from cryopreserved eggs fed on Plasmodium falciparum (Pf)-infected blood meals was similar to that of mosquitoes routinely infected at our facility; the prevalence of infection (50\%) was lower but infection intensities were comparable to our routine infections. Adult female mosquitoes derived from cryopreserved eggs were fed on uninfected blood and a second generation of females then fed on Pf-infected blood-the prevalence of Pf infection in this case reached 93\%. This was not intended to be a controlled experiment: the purpose was to determine whether different generations of mosquitoes reared from cryopreserved eggs could still be infected with Plasmodium falciparum.

We present here, a method that reproducibly yields $\sim 25 \%$ hatched first instar larvae of $A$. stephensi mosquitoes from cryopreserved eggs. The critical components of the technique are the time of harvesting the eggs after laying (15-30 $\mathrm{min})$, the use of absolute $(24.8 \mathrm{M})$ methanol as the CPA, the two-step exposure to CPA $\left(-7^{\circ} \mathrm{C}\right.$ for 6.5-7 min followed by $-14.5^{\circ} \mathrm{C}$ for $\left.\sim 15 \mathrm{~min}\right)$, rapid cooling into $\mathrm{LN}_{2}\left(>-12,240^{\circ} \mathrm{C} \mathrm{min}^{-1}\right)$, and rapid thawing $\left(+30,000^{\circ} \mathrm{Cmin}^{-1}\right)$ with CPA dilution $\left(1: 500\right.$ in water at $\left.23^{\circ} \mathrm{C}\right)$. The method is improved marginally by using D4-methanol and chilled $\mathrm{LN}_{2}$. No pretreatment to permeabilize the egg chorion is required, although surface water is removed before adding the eggs to CPA. To date this method has been successful with all strains of $A$. stephensi tested ${ }^{11,12}$ including a fragile LRIM1 knock out transgenic line ${ }^{30,31}$ and also with A. gambiae (Keele) in one trial experiment (data not shown). We anticipate that the method should work with newly colonized strains.

Received: 16 October 2021; Accepted: 10 December 2021

Published online: 07 January 2022

\section{References}

1. Almeida, A. P. G. \& Billingsley, P. F. Induced immunity against the mosquito Anopheles stephensi Liston (Diptera : Culicidae): effects on mosquito survival and fecundity. Int. J. Parasitol 28, 1721-1731 (1988).

2. Liu, X. H. \& Mazur, P. Effects of sugars on the kinetics of drying and on the survival of partially dehydrated larvae of Anopheles mosquitoes. J. Insect Physiol. 49, 685-695 (2003).

3. Shililu, J. I. et al. Development and survival of Anopheles gambiae eggs in drying soil: influence of the rate of drying, egg age, and soil type. J. Am. Mosq. Control Assoc. 20, 243-247 (2004).

4. Hoffman, S. L. et al. Development of a metabolically active, non-replicating sporozoite vaccine to prevent Plasmodium falciparum malaria. Hum. Vaccin. 6, 97-106 (2010).

5. Epstein, J. E. et al. Live attenuated malaria vaccine designed to protect through hepatic $\mathrm{CD} 8^{+} \mathrm{T}$ cell immunity. Science 34, 475-480 (2011).

6. Seder, R. A. et al. Protection against malaria by intravenous immunization with a nonreplicating sporozoite vaccine. Science 341, 1359-1365 (2013).

7. Lyke, K. E. et al. Attenuated PfSPZ Vaccine induces strain-transcending T cells and durable protection against heterologous controlled human malaria infection. Proc. Natl Acad. Sci. USA 114, 2711-2716 (2017).

8. Mordmüller, B. et al. Sterile protection against human malaria by chemoattenuated PfSPZ vaccine. Nature 542, 445-449 (2017).

9. Mwakingwe-Omari, A. et al. Two chemoattenuated PfSPZ malaria vaccines induce sterile hepatic immunity. Nature 595, 289-294 (2021).

10. Feldman, A. M. \& Ponnudurai, T. Selection of Anopheles stephensi for refractoriness and susceptibility to Plasmodium falciparum. Med. Vet. Entomol. 3, 141-145 (1989).

11. O’Brochta, D. A. et al. Is Saglin a mosquito salivary gland receptor for Plasmodium falciparum?. Malaria J. 18, 2 (2019).

12. Li, T. et al. Robust, reproducible, industrialized, standard membrane feeding assay for assessing the transmission blocking activity of vaccines and drugs against Plasmodium falciparum. Malaria J. 14, 150 (2015). 
13. Farnesi, L. C., Menna-Barreto, R. F. S., Martins, A. J., Valle, D. \& Rezende, G. L. Physical features and chitin content of eggs from the mosquito vectors Aedes aegypti, Anopheles aquasalis and Culex quinquefasciatus: Connection with distinct levels of resistance to desiccation. J. Insect Physiol. 83, 43-52 (2015).

14. Rezende, G. L. et al. Embryonic desiccation resistance in Aedes aegypti: presumptive role of the chitinized serosal cuticle. BMC Dev. Biol. 8, 82. https://doi.org/10.1186/1471-213X-8-82 (2008).

15. Vargas, H. C. M., Farnesi, L. C., Martins, A. J., Valle, D. \& Rezende, G. L. Serosal cuticle formation and distinct degrees of desiccation resistance in embryos of the mosquito vectors Aedes aegypti, Anopheles aquasalis and Culex quinquefasciatus. J. Insect Physiol. 62, 54-60 (2014).

16. Farnesi, L. C., Vargas, H. C. M., Valle, D. \& Rezende, G. L. Darker eggs of mosquitoes resist more to dry condition: Melanin enhances serosal cuticle contribution in egg resistance to desiccation in Aedes, Anopheles, and Culex vectors. PLOS Negl. Trop. Dis. 11, e0006063 (2017).

17. Mazur, P., Cole, K. W., Hall, J. W., Schreuders, P. D. \& Mahowald, A. P. Cryobiological preservation of Drosophila embryos. Science 258, 1932-1935 (1992).

18. Steponkus, P. L. et al. Cryopreservation of Drosophila melanogaster embryos. Nature 345, 170-172 (1990).

19. Liu, X. H., Pan, H. \& Mazur, P. Permeation and toxicity of ethylene glycol and methanol in larvae of Anopheles gambiae. J. Exp. Biol. 206, 2221-2228 (2003).

20. Monnerat, A. T. et al. Anopheles albitarsis embryogenesis: morphological identification of major events. Mem. Inst. Oswaldo Cruz. 97, 589-596 (2002).

21. Morris, G. J. \& Canning, C. E. The cryopreservation of Euglena graciis. J. Gen. Microbiol. 108, 27-31 (1978).

22. Kapoore, R. V. et al. Effects of cryopreservation on viability and functional stability of an industrially relevant alga. Sci. Rep. 9, 2093 (2019).

23. James, E. R. \& Farrant, J. Recovery of infective Schistosoma mansoni schistosomula from liquid nitrogen: A step towards storage of a live schistosomiasis vaccine. Trans. R. Soc. Trop. Med. Hyg. 71, 498-500 (1977).

24. James, E. R. Cryopreservation of Schistosoma mansoni schistosomula using $40 \% \mathrm{v} / \mathrm{v}(10 \mathrm{M})$ methanol and rapid cooling. Cryo-Lett. 1, 535-544 (1980).

25. Smith, B.S., Hodgson, A., Popiel, I., Minter, D. \& James, E.R. Cryopreservation of steinernematid entomogenous nematodes. Proceedings of the 2nd meeting of the I.O.B.C./W.P.R.S. Working Group on "Insect Pathogens and Entomoparasitic Nematodes," Rome, Italy, 6-8 (1989).

26. Anderson, S. A., Hulston, D. A., McVeagh, S. M., Webb, V. L. \& Smith, P. J. In vitro culture and cryopreservation of Uronema marinum isolated from farmed New Zealand groper (Polyprion oxygeneios). J. Microbiol. Methods. 79, 62-66 (2009).

27. Guan, M., Rawson, D. M. \& Zhang, T. Cryopreservation of zebrafish (Danio rerio) oocytes using improved controlled slow cooling protocols. Cryobiol. 56, 204-208 (2008).

28. Evans et al. Quantitative interpretation of diffusion-ordered NMR spectra: Can we rationalize small molecule diffusion coefficients. Supporting information [https://onlinelibrary.wiley.com/action/downloadSupplement?doi $=10.1002 \% 2$ Fanie $201207403 \&$ file= anie_201207403_sm_miscellaneous_information.pdf], Angew. Chem. Int. Ed.Engl, 52, 3199-3202 (2013).

29. Whittingham, D. C., Leibo, S. P. \& Mazur, P. Survival of mouse embryos frozen to -196 degrees and -269 degrees C. Science 178, 411-414 (1972).

30. Rall, W. F. \& Wood, M. J. High in vitro and in vivo survival of day 3 mouse embryos vitrified or frozen in a non-toxic solution of glycerol and albumin. J. Reprod. Fertil. 101, 681-688 (1994).

31. Inbar, E. et al. Knockout of Anopheles stephensi immune gene LRIM1 by CRISPR-Cas9 reveals its unexpected role in reproduction and vector competence. PLoS Pathog. 17, e10009770 (2021)

32. Billingsley, P. F. et al. Transient knockdown of Anopheles stephensi LRIM1 using RNAi increases Plasmodium falciparum sporozoite salivary gland infections. Malaria J. 20, 1-12 (2021).

\section{Acknowledgements}

We thank the Sanaria Gametocyte Production Team and the Vaccine Extraction and Immunology Team for providing infectious bloodmeals and for assessment of infections of Pf in mosquitoes.

\section{Author contributions}

Conceptualization: E.R.J. Methodology: E.R.J., Y.W., J.O., K.P., S.M.cT., S.M. Funding acquisition: P.F.B., E.R.J., S.L.H. Project administration: P.F.B., E.R.J. Supervision: E.R.J., P.F.B., A.E. Writing_original draft: E.R.J. Writing-review and editing: E.R.J., P.F.B., S.L.H.

\section{Funding}

This article was funded by National Institutes of Health (grant R44AI088853).

\section{Competing interests}

ERJ: Patent pending US2020/041854, International Publication Number: WO/2021/011503. YW, JO, KP, SMcT, SM, AE, SLH, PFB: No competing interests.

\section{Additional information}

Supplementary Information The online version contains supplementary material available at https://doi.org/ 10.1038/s41598-021-04113-X.

Correspondence and requests for materials should be addressed to E.R.J.

Reprints and permissions information is available at www.nature.com/reprints.

Publisher's note Springer Nature remains neutral with regard to jurisdictional claims in published maps and institutional affiliations. 
(c) (i) Open Access This article is licensed under a Creative Commons Attribution 4.0 International cc) License, which permits use, sharing, adaptation, distribution and reproduction in any medium or format, as long as you give appropriate credit to the original author(s) and the source, provide a link to the Creative Commons licence, and indicate if changes were made. The images or other third party material in this article are included in the article's Creative Commons licence, unless indicated otherwise in a credit line to the material. If material is not included in the article's Creative Commons licence and your intended use is not permitted by statutory regulation or exceeds the permitted use, you will need to obtain permission directly from the copyright holder. To view a copy of this licence, visit http://creativecommons.org/licenses/by/4.0/.

(C) The Author(s) 2022 